\title{
COMPASS Polarized Target For Drell-Yan Experiment
}

\author{
J. Matoušek and M. Pešek \\ Faculty of Mathematics and Physics, Charles University in Prague, Czech Republic \\ E-mail: jan.matousek@cern.ch, michael.pesek@cern.ch
}

In the COMPASS Drell-Yan experiment the pion beam with momentum of $190 \mathrm{GeV} / \mathrm{c}$ and intensity up to $10^{8}$ pions/s will interact with transversely polarized proton target producing muon pair via Drell-Yan process. The solid-state $\mathrm{NH}_{3}$ will be polarized by dynamic nuclear polarization. Maximum polarization reached during data taking is expected to be up to $90 \%$. The non-interacting beam and other particles produced inside the target will be stopped in the hadron absorber after the target. Two target cells, sepparated by a $20 \mathrm{~cm}$ gap in between, each $55 \mathrm{~cm}$ long and $4 \mathrm{~cm}$ in diameter give the target material volume about $691 \mathrm{~cm}^{3}$.

The target platform needs to be moved by $2.3 \mathrm{~m}$ in upstream direction from the position used in previous experiments in order to accomodate the absorber. During the beam time higher radiation is expected in the area of the control room. Thus a new target remote control system is needed. The target magnet is undergoing a substantial upgrade.

Drell-Yan data taking is expected to start in 2014-2015 for period of approximately 180 days. Current status of the target, the required modifications and future plans are presented.

XVth International Workshop on Polarized Sources, Targets, and Polarimetry, September 9-13, 2013

Charlottesville, Virginia, USA

\footnotetext{
*Speaker.

On behalf of the COMPASS Collaboration.
} 


\section{Introduction}

COmmon Muon and Proton Apparatus for Structure and Spectroscopy (COMPASS) [3] is a fixed-target experiment situated at CERN Super Proton Synchrotron (SPS) North Area. For physics data taking it uses hadron or muon beam ${ }^{1}$, which can be both positive or negative with momentum up to $280 \mathrm{GeV} / c$. Muons are naturally longitudinally polarized. The beam interacts with a target, which can be both polarized and unpolarized. COMPASS detector is a universal two-stage spectrometer, each stage (Large Angle Spectrometer and Small Angle Spectrometer) based on the dipole magnet, equipped with a sufficient number of tracking detector planes and electromagnetic and hadron calorimeters. There is a Ring Imaging Cherenkov (RICH) detector too, which is an efficient tool for particle identification.

Since its approval in 1997 COMPASS experiment at CERN focused at spin structure studies and hadron spectroscopy using variety of targets [1]. A set of measurements to study the structure of hadrons was proposed [2], including the first ever measurement of a single-polarized DrellYan (DY) process using a pion beam and a transversely-polarized proton target. It aims to confirm some crucial predictions of QCD, namely universality i.e. change of sign of the Sivers and BoerMulders TMDs when measured in Semi-Inclusive Deep Inelastic Scattering (SIDIS) and in DY processes.

As the DY cross section is low, the luminosity should be as high as possible. In COMPASS case this corresponds to the beam intensity of about $10^{8}$ pions/s. That is the highest hadron beam intensity COMPASS has ever used, which leads to several challenges for the detection, data acquisition and polarized target.

\section{Polarized target}

The Polarized Target (PT) system [3, 4] has an important role in COMPASS spin structure studies. It can provide large amount ${ }^{2}$ of target material polarized to a high degree ${ }^{3}$. The material is polarized by Dynamic Nuclear Polarization (DNP) method [6] at about $0.5 \mathrm{~K}$. When the optimal polarization is reached, the target is switched to a "frozen spin" mode at about $50 \mathrm{mK}$. A long spinlattice relaxation time at such temperature (in order of $10^{3}$ hours) allows to perform reasonably efficient experiment. Two Microwave (MW) systems for DNP allow to have target cells with opposite polarization to reduce systematic errors in measured asymmetries. The magnetic field rotation, performed normally on a day-by-day basis, serves the same purpose. The key elements of the PT are: a Dilution Refrigerator (DR), which has a cooling power of about $5 \mathrm{~mW}$ at $75 \mathrm{mK}$ [7]; a large-aperture superconducting magnet, which can provide a field of $2.5 \mathrm{~T}$ parallel and $0.64 \mathrm{~T}$ perpendicular to the beam axis; MW system and a continuous-wave NMR system for polarization measurement. The homogeneity of the magnetic field is about $10^{-5} \mathrm{~T}$.

During 2010-2011 the COMPASS experiment was mostly running with muon beam. The 3cell configuration of the PT was used $(30-60-30 \mathrm{~cm}, 4 \mathrm{~cm}$ in diameter). The target material was a solid-state $\mathrm{NH}_{3}$ (previously irradiated by an electron beam to induce paramagnetic centers for

\footnotetext{
${ }^{1}$ Produced by proton beam from SPS hitting a Be target.

${ }^{2} \mathrm{~A}$ cylinder of about $4 \mathrm{~cm}$ in diameter and about $120 \mathrm{~cm}$ long

${ }^{3}$ Over $80 \%$ in case of $\mathrm{H}$ in $\mathrm{NH}_{3}$ and over $50 \%$ in case of $\mathrm{D}$ in ${ }^{6} \mathrm{LiD}$ [5]
} 


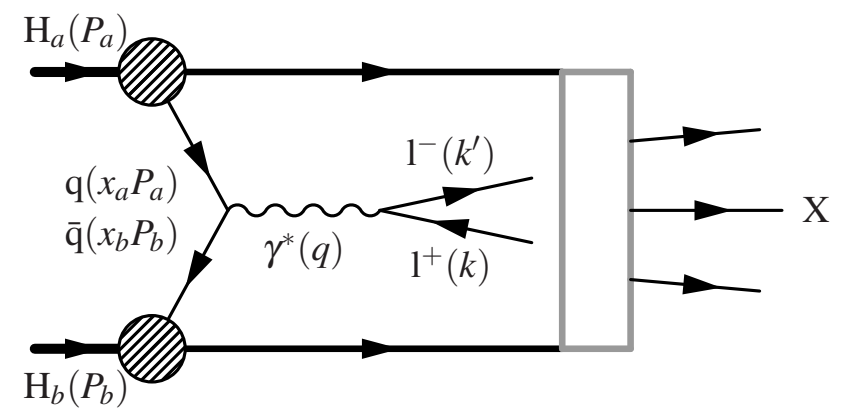

Figure 1: The Drell-Yan process. A quark-antiquark pair from the two hadrons annihilate, producing a lepton-antilepon pair in final state. The grey box denotes hadronization.

DNP). The MW cavity had two $5 \mathrm{~cm}$ long MW stoppers to allow opposite polarization in the middle cell and the two outer cells. The NMR system consisted of 10 NMR coils ( 3 for each outer cell, 4 for the middle cell). The target operated in transverse-polarization mode during run 2010 and in longitudinal-polarization mode during run 2011. Average maximum polarization of about $83 \%$ was achieved [8]. The main limitation for the maximum polarization was the time available for polarizing the target.

\section{Drell-Yan program at COMPASS}

Drell-Yan (DY) process can be represented by Feynman graph on Fig. 1. The main advantage of DY process for hadron structure studies is that there are leptons in the final state, which means that the cross section does not involve any fragmentation function but only convolution of structure functions of both hadrons. The process is very well calculable, a dedicated calculations of the pioninduced DY process for the COMPASS kinematics were recently published [9]. The disadvantage of the DY process is a low cross section.

As was already mentioned, the high intensity pion beam will be used resulting in a large secondary-hadron flux. The non-interacting beam and all secondary particles except muons should be stopped right after the target to avoid a spectrometer flooding-up [2]. A special hadron absorber was designed for this purpose and is being produced. It will be made of stainless steel and alumina, with a tungsten beam plug in the center to stop the beam. For the measurement at COMPASS this means worse vertex resolution in comparison with SIDIS program, as the absorber introduces significant multiple scattering. A dedicated di-muon trigger has to be designed.

The high intensity pion beam together with the hadron absorber will cause higher radiation dose $^{4}$ in the experimental building. Because of that the control room will be moved to another building.

\section{Modifications of the PT for the DY program}

The intensive pion beam and presence of the hadron absorber brings need for modifications of PT. Because of the absorber the whole target have to be moved $2.3 \mathrm{~m}$ upstream from the standard (SIDIS) position. Given the worse vertex resolution, a wider gap between the target cells is needed

\footnotetext{
${ }^{4}$ About $3.6 \mu \mathrm{Sv} / \mathrm{h}$, which exceeds CERN limit for permanently occupied area
} 


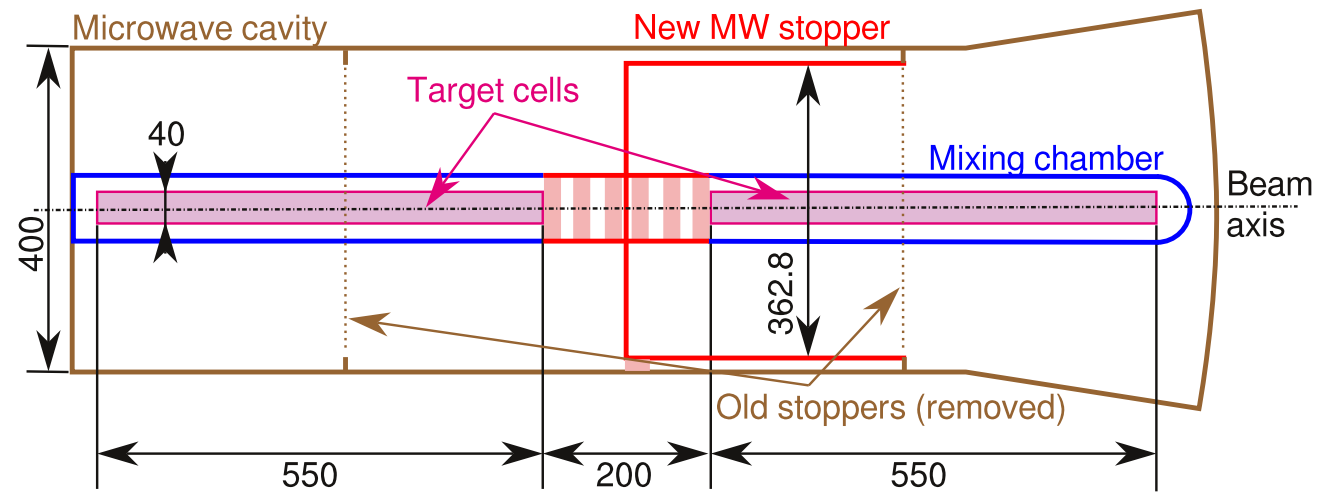

Figure 2: The modified MW cavity. The upstream MW stopper was removed, the downstream one was replaced by a special adapter and a wider stopper fitting in the $20 \mathrm{~cm}$ gap between the $55 \mathrm{~cm}$ long target cells.

to ensure correct assignment of events to the cells. The beam spot should be larger to prevent local overheating of the target by the intensive beam.

The new cell design consists of two cells ( $4 \mathrm{~cm}$ in diameter, $55 \mathrm{~cm}$ long) with $20 \mathrm{~cm}$ long MW stopper. The wider space between oppositely polarized cells will help to properly distinguish primary vertices coming from the two cells. The NMR system will probably consist of 10 NMR coils. 4 coils will be placed outside of each cell (for measurement in longtudinal field) and one inside each cell (coil axis parallel to the beam axis-for measurement in transverse field). Design of the coils is in development. A special adapter was designed for the MW cavity to accommodate one MW stopper of $20 \mathrm{~cm}$ length instead of two (see the Fig. 2).

The target platform will be lifted by a crane and moved to its new position $2.3 \mathrm{~m}$ upstream. A special support craning construction was designed by CERN for this purpose. The helium lines, cabling, MW waveguides and other equipment will be moved to the new position as well.

It was decided to abandon the LabVIEW ${ }^{\mathrm{TM}}$ system [10] for dilution refrigerator monitoring. A new, more robust, Linux-based software package called ptread is being developed instead. It is written $\mathrm{C}++$ and Perl. It can communicate with the standard COMPASS DCS ${ }^{5}$ via DIM library [11] and write data into MySQL or SQLite database. These features are important for the remote monitoring ${ }^{6}$. The main advantage is that the package is modular and easily adjustable. The target configuration can now be loaded from a file, so it is easier to e. g. exchange an instrument. Fig. 3 shows functionality of the monitoring system.

The superconducting target magnet is being refurbished by CERN magnet group. The work is almost finished. The vacuum was successfully tested recently and cooling tests will follow soon. The group will provide control and safety systems too.

\footnotetext{
${ }^{5}$ Centralized, PVSS-based detector control system

${ }^{6}$ Necessary, since the control room will be moved, as was mentioned before.
} 


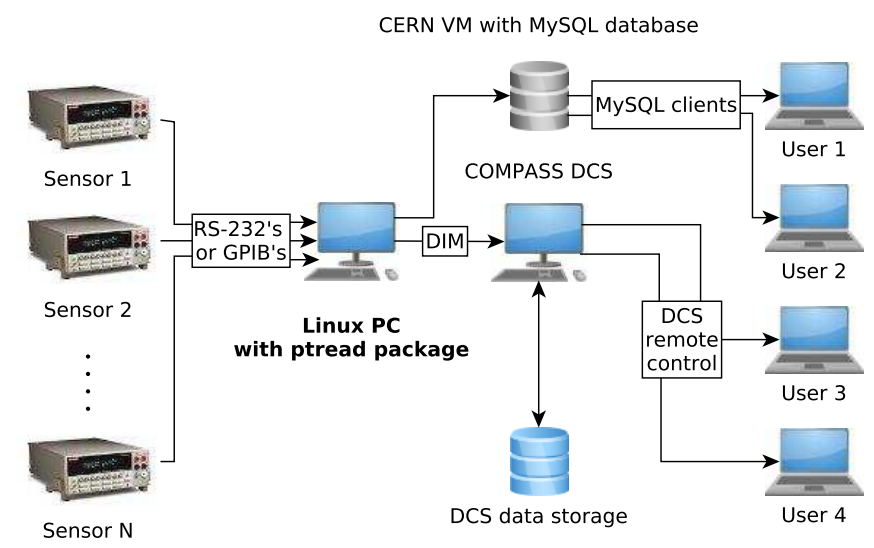

Figure 3: Diagram of ptread communication. The Linux computer with ptread package reads sensors. Data can be stored locally in SQLite database, sent to MySQL database and published by DIM server for COMPASS DCS.

\section{Status of the polarized target}

The modified MW cavity was successfully tested earlier this year. The technical drawing of the new target cells is being finished and the production of the cells is expected in the near future. The movement of the target platform is planned to be done by the end of 2013. The superconducting magnet refurbishment is finishing, the magnet should be ready for installation in February 2014. The dilution refrigerator was leak-tested at room temperature, no leaks were found.

\section{Conclusion}

COMPASS has now possibility to make unique measurement of single-polarized Drell-Yan process. It will be the first ever study of a polarized DY. Preparation for this involves polarized target modifications which are progressing well. The target should be ready and fully operational by the end of next summer to allow measurement during the fall 2014 (after CERN long shutdown of accelerators) and over the whole 2015. 


\section{Acknowledgments}

The authors acknowledge support of HP3 EU, MEYS LA08015 and SVV-2013-267303 Czech Republic grants.

\section{References}

[1] The COMPASS Collaboration, List of COMPASS publications, http: / / wwwcompass.cern.ch/compass/publications /

[2] The COMPASS Collaboration, COMPASS-II Proposal, CERN-SPSC-2010-014, SPSC-P-340.

[3] P. Abbon et al., The COMPASS experiment at CERN, NIM A 577 (2007) 455-518.

[4] J. H. Koivuniemi et al., Journal of Physics: Conference series 150 (2009) 012023.

[5] K. Kondo et al., Polarization measurement in the COMPASS polarized target, NIM A 526 70-75.

[6] A. Abragam, M. Goldman, Principles of dynamic nuclear polarisation, Rep. Prog. Phys. 41 (1978) 395.

[7] N. Doshita et al., Performance of the COMPASS polarized target dilution refrigerator, NIM A 526 (2004) 138-143.

[8] M. Mešek, Low temperature proton polarized target for nucleon structure studies at COMPASS, bachelor thesis, Charles university in Prague (2012), http:

//wwwcompass.cern.ch/compass/publications/theses/2012_bac_pesek.pdf.

[9] M. Aicher, A. Schäfer and W. Vogelsang, Threshold-resumed cross section for the Drell-Yan process in pion-nucleon collisions at COMPASS, Phys. Rev. D 83 (2011) 114023.

[10] F. Gautheron et al., Cryogenic control system of the large COMPASS polarized target, NIM A 526 (2004) 147-152.

[11] C. Gaspar et al., Distributed information management system, http://dim.web.cern. ch/dim/. 